\title{
Rotational Atherectomy in Acute ST-Elevation Myocardial Infarction
}

\author{
Ann Kee How; ${ }^{1 \star}$ Kamaraj Selveraj, ${ }^{1}$ Abdul Kahar Abdul Ghapar ${ }^{1}$ \\ 'Cardiology Department, Hospital Serdang, Selangor, Malaysia \\ * Corresponding author. Email: akhow_82@yahoo.com; Address: Cardiology Department, Hospital Serdang, Jalan Puchong, 43000 Kajang, Selangor, Malaysia
}

\begin{abstract}
Rotational atherectomy or rotablation is commonly used in lesion preparation for calcified coronary artery. However, rotablation is relatively contraindicated in cases with high thrombus load lesion, such as acute ST-elevation myocardial infarction (STEMI). We are presenting a case of acute STEMI treated with rotablation. Rotablation was used to facilitate the primary percutaneous coronary intervention (PCl) with calcified coronary artery, after unsuccessful attempt with scoring balloon dilation. Although there was slow flow due to high thrombus load in STEMI, we learnt that with meticulous rotablation techniques, the procedure could be done safely. The final result was excellent and patient was discharged well after 3 days.
\end{abstract}

\section{INTRODUCTION}

Coronary artery calcification is associated with adverse outcomes following $\mathrm{PCl}$ and coronary intervention of calcified coronary is indeed very challenging. The challenges commonly faced are: difficulty in wiring the lesion, delivering balloon or stent and possibility of stent under-expansion with subsequent risk of stent thrombosis. Therefore, it is mandatory to prepare the lesion well prior to stent implantation. Rotational atherectomy or rotablation is a useful tool for plaque modification in calcified coronary, as a sole therapy or adjunct to scoring or cutting balloon. Atherectomy can be done safely in myocardial infarction patients. Lee MS, et al. (2017) showed that the primary endpoint was similar in patients who presented with and without non-ST-elevation myocardial infarction (NSTEMI) who underwent orbital atherectomy. ${ }^{4}$ The 30-day rates of death, myocardial infarction, target vessel revascularization and stroke were similar in both groups. ${ }^{4}$

\section{CAse presentation}

A 53-year-old patient presented with severe chest pain and acute anterior STEMI recorded on electrocardiogram (Figure 1), was referred for primary $\mathrm{PCl}$. Patient had underlying diabetes mellitus, hypertension and dyslipidaemia. On arrival to our unit, patient was stable haemodynamically with on-going chest pain. Patient was loaded with oral Aspirin $300 \mathrm{mg}$ and Ticagrelor 180mg.

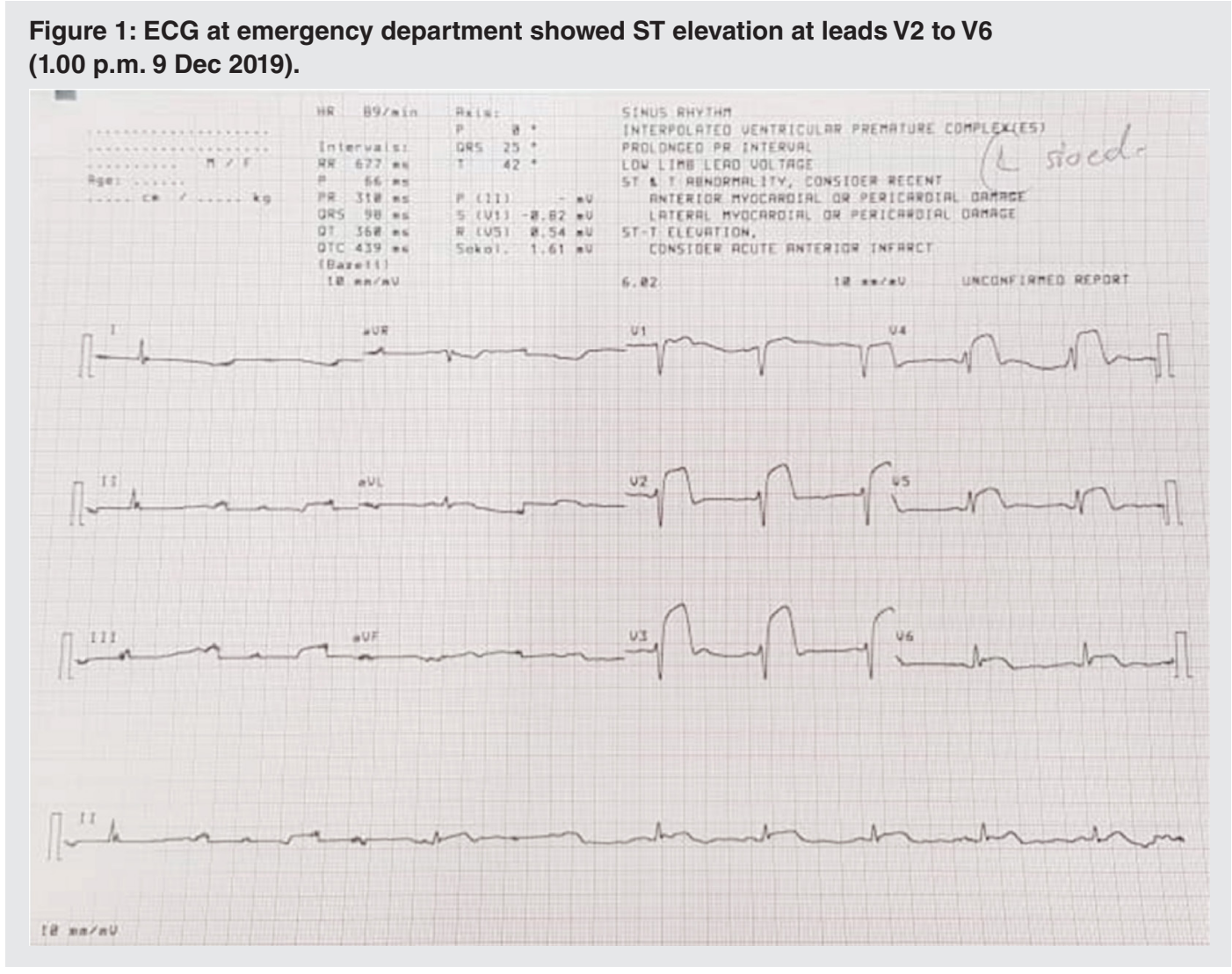


Coronary angiogram was performed via right radial access with 6 -French sheath. The left main was smooth. There was severe left anterior descending (LAD) artery calcification with proximal LAD 80\% stenosis followed by mid LAD acute total occlusion (ATO), thrombolysis in myocardial infarction (TIMI) 0 flow (Figure 2). The left circumflex (LCX) artery was small with distal LCX 80 $90 \%$ diffuse disease. Right coronary artery (RCA) was dominant, minor disease with distal posterior left ventricular (PLV) and distal posterior descending artery (PDA) 90\% disease.

\section{Figure 2: Coronary angiogram showed severe LAD artery calcification with proximal LAD $\mathbf{8 0} \%$ stenosis, followed by mid LAD ATO (1.15 p.m. 9 Dec 2019).}

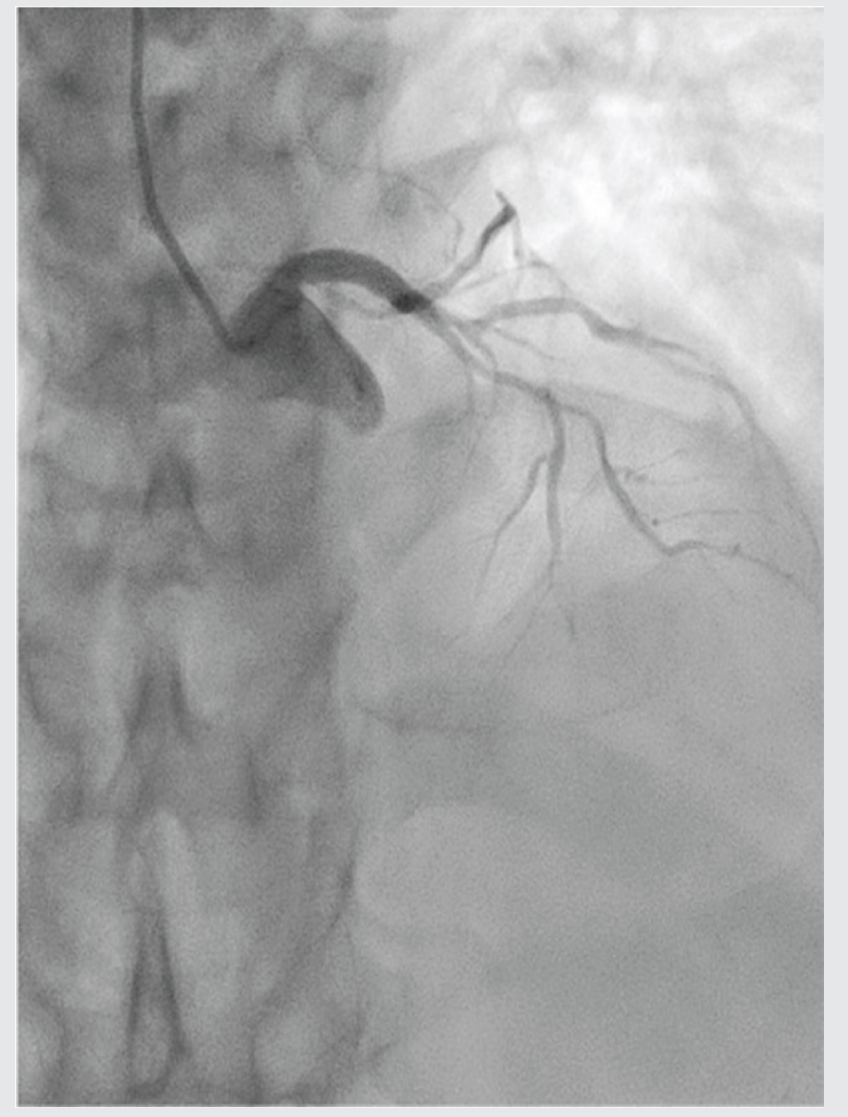

$\mathrm{PCI}$ to $\mathrm{LAD}$ was performed with radial approach and 6-French Extra Back-up (EBU) 3.0 guiding catheter. LAD was wired with Sion Blue (ASAHI) and aspirated with Export Advance aspiration catheter (Medtronic). The lesion was crossed with minimal difficulty. This was followed by pre-dilation with $2.5 \times 15-\mathrm{mm}$ non-compliant (NC) balloon. The balloon was under-expanded at the calcified segment (Figure 3). Further pre-dilation with scoring balloon $2.5 \times 15-\mathrm{mm}$ also showed under-expanded balloon at the same calcified segment.

Patient had significant on-going chest pain despite improved coronary flow. We decided to proceed with rotational atherectomy (Rotablator, Boston Scientific) similarly via radial approach, to debulk the calcified lesion. Finecross (Terumo) micro-catheter was used to exchange to a Rotafloppy wire (Boston Scientific). The rotablation was performed with a 1.5$\mathrm{mm}$ burr at 160,000 rotations per minute (rpm) (Figure 4). Burr time and rotational speed deceleration were kept at less than 15 seconds and $5000 \mathrm{rpm}$, respectively. There was slow flow post rotablation due to high thrombus load in acute STEMI.
Figure 3: Coronary angiogram showed under-expanded 2.5mm NC balloon at calcified segment (1.25 p.m. 9 Dec 2019).

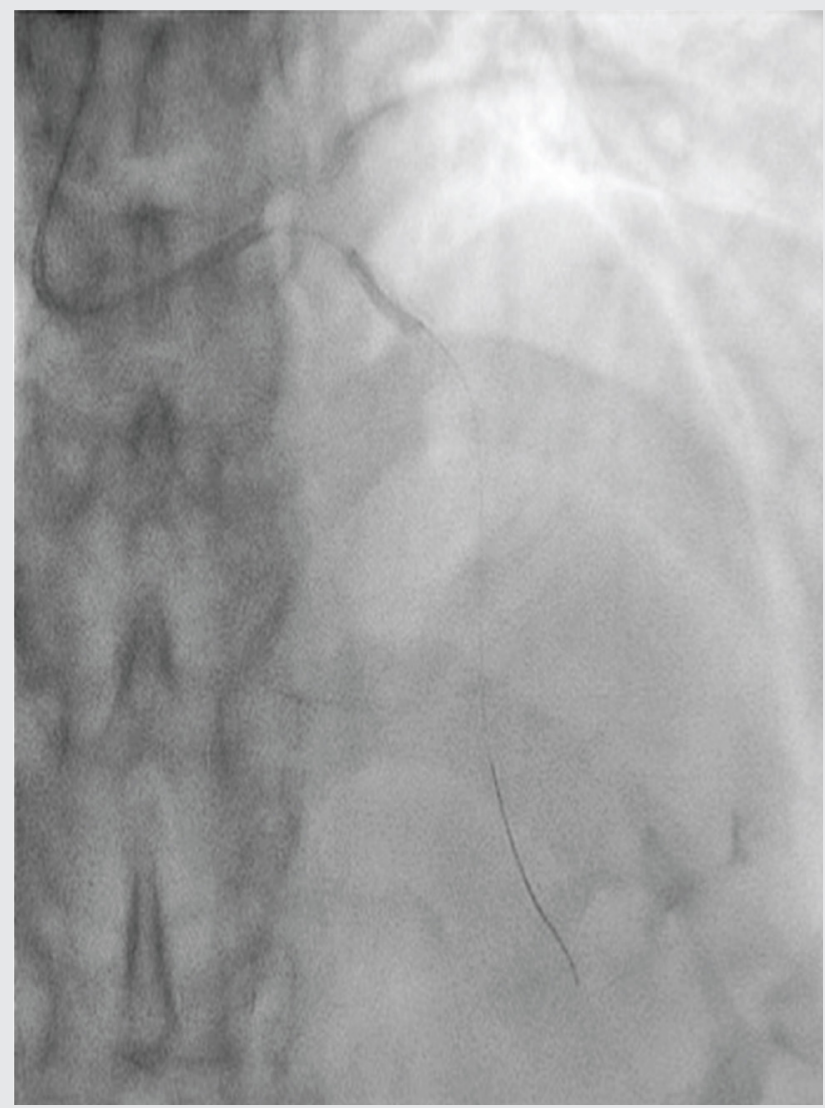

Figure 4: Rotablation of the LAD artery with $1.5-\mathrm{mm}$ burr (1.40 p.m. 9 Dec 2019).

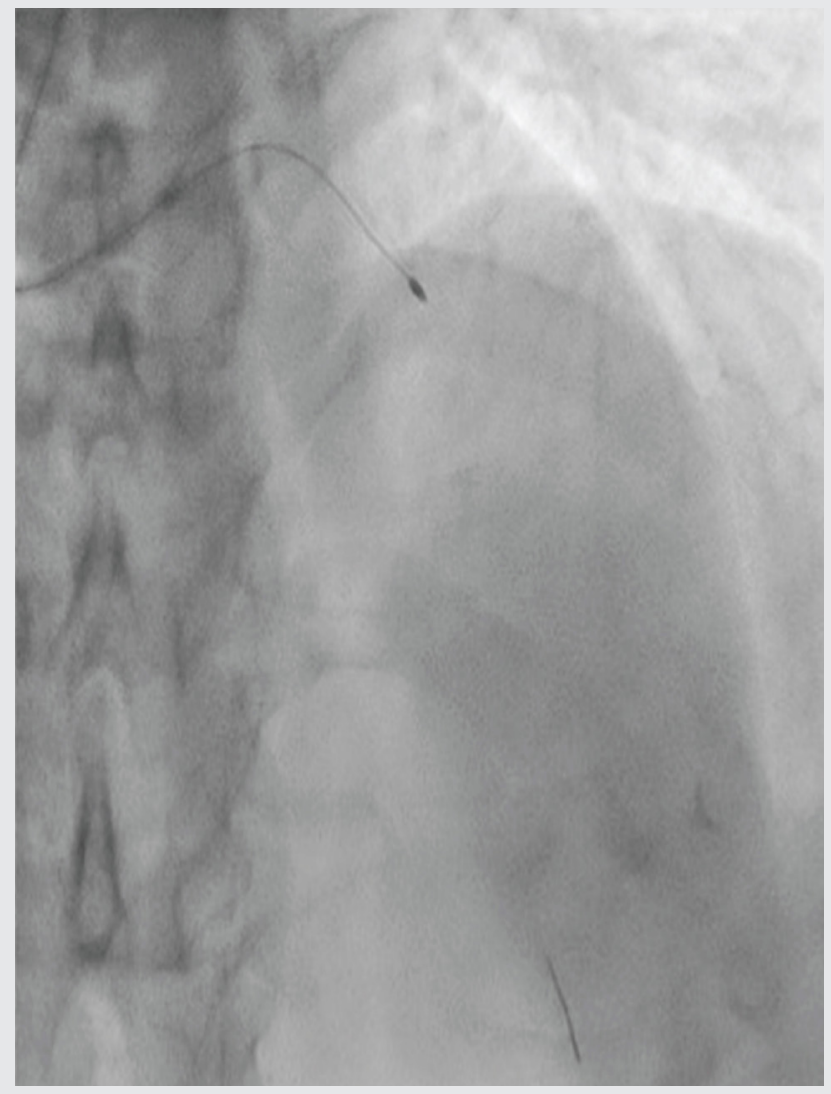


Fortunately, patient was stable haemodynamically and the slow flow was transient. The LAD lesion was then further pre-dilated with 3.0-mm NC balloon and the balloon expanded well.

Two drug-eluting stents (DES) were used, a $2.5 \times 38$-mm DES was deployed at mid LAD, followed by $3.0 \times 34-\mathrm{mm}$ DES at ostial to proximal LAD. A 3.5-mm NC balloon was used to post-dilate the stent, establishing TIMI 3 flow and excellent angiographic result (Figure 5)

\section{Figure 5: Final angiographic result with well-expanded stents (2.10 p.m. 9 Dec 2019).}

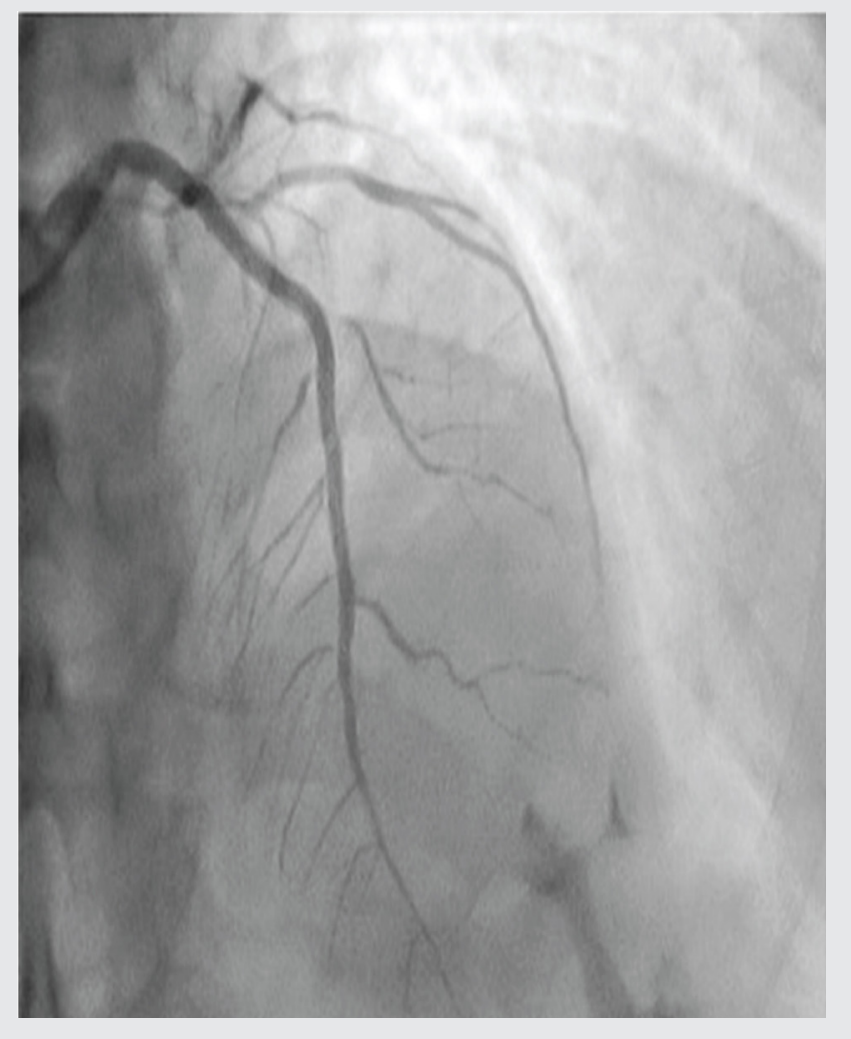

The total time used for the $\mathrm{PCl}$ was 65 minutes with first medical contact (FMC) to reperfusion time of 37 minutes. Echocardiogram showed ejection fraction (EF) 55\%, hypokinetic mid anteroseptal and apical anterior wall with normal valves. There was no periprocedural complication and the patient was discharged well after 3 days. Patient was indeed very grateful that the revascularization procedure was safely done despite technical challenge encountered.

\section{DISCUSSION}

Conventionally, the use of rotational atherectomy should be avoided in the presence of thrombus due to the concern of further platelet activation and aggregation by the spinning rotablator burr. ${ }^{1}$ Therefore, option like thrombolytics are considered as initial treatment with subsequent rotational atherectomy two to four weeks later. ${ }^{3}$

Slow flow or no reflow during rotational atherectomy is closely related to microvascular embolization of atherosclerotic debris and thrombi, platelet activation and aggregation or microcirculatory vasospasm. Strategies to prevent these problems include optimal antiplatelet therapy, meticulous interventional techniques and the use of vasodilators. ${ }^{2}$ Atheromatous debris embolism can be avoided by appropriate burr size, gradual burr advancement, short ablation runs and minimal decelerations. Optimal antiplatelet therapy including use of glycoprotein Ilb/llla inhibitor and slower rotational speed can prevent platelets aggregation associated with rotational atherectomy. The use of vasodilators, including adenosine, calcium antagonists and nitroglycerin can be considered in slow flow due to microcirculatory vasospasm and to enhance microvascular clearance.

In the presence of severe calcification and improved coronary flow after initial pre-dilation with non-compliant and scoring balloons, the option to defer rotational atherectomy can be considered. We decided not to delay the procedure as patient had on-going chest pain. We had chosen a 1.5-mm burr, which was considered appropriate size for the artery, ran at relative low rotational speed and short burr time. This was to minimize distal embolization and prevent dissection that may worsen the slow flow. With all these meticulous steps, the slow flow was fortunately transient and subsequent procedure was uneventful. Allali A et al. (2019) and Shahin M, et al. (2018), also reported that rotational atherectomy was feasible and safe in high-risk patients presenting with ACS. 5,6

\section{CONCLUSIONS}

Although we do not recommend the routine use of rotational atherectomy for calcified coronary intervention in acute STEMI, it can be considered for cases with strong clinical indication when standard interventions fail. In our case, we encountered a calcified undilatable lesion, which was amendable with rotational atherectomy. Our case illustrates that with modern armamentarium of devices and pharmacology, the nonconventional approaches become possible and safe.

\section{KEYWORDS}

Rotational atherectomy, calcification, acute myocardial infarction

\section{REFERENCES}

1. Ho PC, Rotational Coronary Atherectomy in Acute ST-segment Elevation Myocardial Infarction, J Interv Cardiol, 18 (4): 315-318, 2005.

2. Tomey MI, Kini AS, Sharma SK, Current status of Rotational Atherectomy JACC Cardiovasc Interv, 7(4): 345-53, April 2014

3. Rotablator ${ }^{\mathrm{TM}}$ Rotational Atherectomy System. Boston Scientific, https:// www.bostonscientific.com/en-US/products/atherectomy-systems/rotationalatherectomy-systems/rotablator/rotablator-indications-safety-and-warnings. html. Accessed 5 April 2020

4. Lee MS, Shlofmitz E, Lluri G, et al. Outcomes of patients with myocardial infarction who underwent orbital atherectomy for severely calcified lesions. Cardiovasc Revasc Med, 18 (7): 497-500, Oct - Nov 2017.

5. Allali A, Abdelghani M, Mankerious N, et al. Feasibility and clinical outcomes of rotational atherectomy in patients presenting with an acute coronary syndrome. Catheter Cardiovasc Interv, 93 (3): 382 - 89, 15 Feb 2019.

6. Shahin M, Candreva A, Siegrist PT, Rotational Atherectomy in Acute STEMI with Heavily Calcified Culprit Lesion is a Rule Breaking Solution, Curr Cardiol Rev, 14: 213-216, 2018. 